\title{
A Importância na Identificação dos Fatores de Risco para Prevenção do Câncer de Mama
}

\author{
Felipe A. de A. Souza \\ Universidade Severino Sombra, Acd. de medicina \\ faandrades@hotmail.com \\ Juliana L. Fernandes \\ Universidade Severino Sombra, Acd. de medicina \\ ju_lobinha@hotmail.com \\ Natália de S. Moraes \\ Universidade Severino Sombra, Acd. de medicina \\ natalia_samoraes@hotmail.com \\ Luiz Francisco F. Noronha \\ Universidade Severino Sombra, Acd. de medicina \\ luizinho44@gmail.com
}

\begin{abstract}
Resumo: O câncer de mama é, provavelmente, o mais temido pelas mulheres, devido a sua elevada freqüencia e, sobretudo, pelos efeitos psicológicos que as afetam, alterando a percepção da sexualidade, auto-estima e imagem pessoal. $\dot{E}$ o segundo tipo de câncer mais freqüente no mundo e o mais comum entre as mulheres. A identificação dos fatores de risco na população feminina constitui uma medida importante para a prevenção e abordagem terapêutica adequada. Foram aplicados questionários sobre fatores de risco para câncer de mama em 266 mulheres. O presente trabalho tem por objetivo a análise dos principais fatores de risco relacionados ao câncer de mama nas mulheres, objetivando a detecção precoce e o encaminhamento ao ginecologista, visando reduzir a taxa de mortalidade e, por muitas vezes, evitando a intervenção cirúrgica.
\end{abstract}

Palavras-chave: Câncer de mama. Fatores de risco. Prevenção.

\section{The Importance in Identification of Risk Factors for Breast Cancer Prevention}

Abstract: Breast cancer is probably the most feared by women, due to its high frequency and, especially, the psychological effects that affect them, altering the perception of sexuality, self-esteem and personal image. It is the second most common cancer worldwide and the most common among women. The identification of risk factors in the female population is an important measure for the prevention and appropriate therapeutic approach. Questionnaires were

Revista de Saúde, Vassouras, v. 2, n. 2, p. 29-36, jul./dez., 2011 
applied on risk factors for breast cancer in 266 women. The present work aims at analyzing the main risk factors related to breast cancer in women, aiming at early detection and referral to a gynecologist in order to reduce the mortality rate, and often avoiding surgery.

Keywords: Breast cancer, risk factors, prevention.

\section{Introdução}

O câncer de mama é, provavelmente, o mais temido pelas mulheres, devido a sua elevada freqüência e, sobretudo, pelos efeitos psicológicos que as afetam, alterando a percepção da sexualidade, auto-estima e imagem pessoal. O câncer de mama é o segundo tipo de câncer mais freqüente no mundo e o mais comum entre as mulheres, e sua incidência vem aumentando ao longo do tempo. A neoplasia maligna de mama é responsável por cerca de $20 \%$ da incidência de câncer e por $14 \%$ do total de mortes associadas às neoplasias, entre as mulheres (Inumaru, 2011).

No Brasil, bem como em outros países da América Latina, onde ainda prevalece o diagnóstico tardio da doença, seria de grande relevância o emprego de uma abordagem englobadora da questão, visando antecipar sua detecção e controle enquanto problema de saúde pública.

Segundo o Instituto Nacional do Câncer (2008), fatores de risco tais como: antecedente pessoal de câncer de mama; história familiar; primeira gestação após os 30 anos; menarca antes dos 12 anos; menopausa após os 55 anos; nuliparidade; tabagismo; sedentarismo; obesidade; radiações ionizantes para outros tratamentos; uso de terapia de reposição hormonal e anticoncepcional hormonal por longos períodos são fatores determinantes para que as mulheres possam desenvolver o câncer de mama.

A densidade mamária também é um importante fator a ser considerado para câncer de mama, mesmo em mulheres sem história familiar da doença. Em geral, mulheres cujo tecido mamário denso conta mais que $60 \%$ a $75 \%$ da mama têm risco aumentado de câncer de mama de quatro a seis vezes em comparação às mulheres com mamas liposubstituídas.

A identificação desses fatores de risco na população feminina constitui uma medida importante para a prevenção e abordagem adequada. Com tal medida é possível ofertar uma maior segurança ao tratamento das mulheres, melhorando de forma substancial a sua qualidade de vida.

O presente trabalho tem por objetivo a análise dos principais fatores de risco relacionados ao câncer de mama nas mulheres, objetivando a detecção precoce e o encaminhamento ao ginecologista, visando reduzir a taxa de mortalidade e, por muitas vezes, evitando a intervenção cirúrgica. 


\section{Materiais e Métodos}

Estudo transversal realizado de base populacional através de questionário preenchido em entrevista por mulheres na faixa etária superior a 15 anos. Para a definição da casuística, utilizou-se como critério a faixa etária citada na literatura.

No questionário foram abordadas perguntas referentes aos principais fatores de risco para o desenvolvimento de câncer de mama: idade, menarca precoce, menopausa tardia, nuliparidade ou primiparidade em idade avançada, história de doença mamária benigna e história familiar de câncer mamário em um ou mais parentes de primeiro grau.

Foram coletadas informações sobre variáveis demográficas, comportamentais, biológicas e referentes ao manejo médico. As entrevistadas faziam parte da demanda espontânea do Hospital Universitário Sul - Fluminense e também da demanda espontânea em feiras de saúde realizadas, no presente ano, na cidade de Vassouras - RJ, perfazendo um total de 266 entrevistas.

As informações foram inseridas em banco de dados do software Epi-Info versão 6.04 . As tabelas e gráficos foram construídos no Microsoft Excel 97. A correlação entre as variáveis foi verificada pelo teste $\mathrm{c} 2$, ao nível de significância $\mathrm{p}=0,05$.

O estudo foi aprovado pela Comissão de Ética da Universidade Severino Sombra e as entrevistadas forneceram consentimento por escrito para participação.

\section{Resultados}

Dos 266 questionários foi observado que 40,6\% das mulheres tinham idade superior a 40 anos (Figura 1).

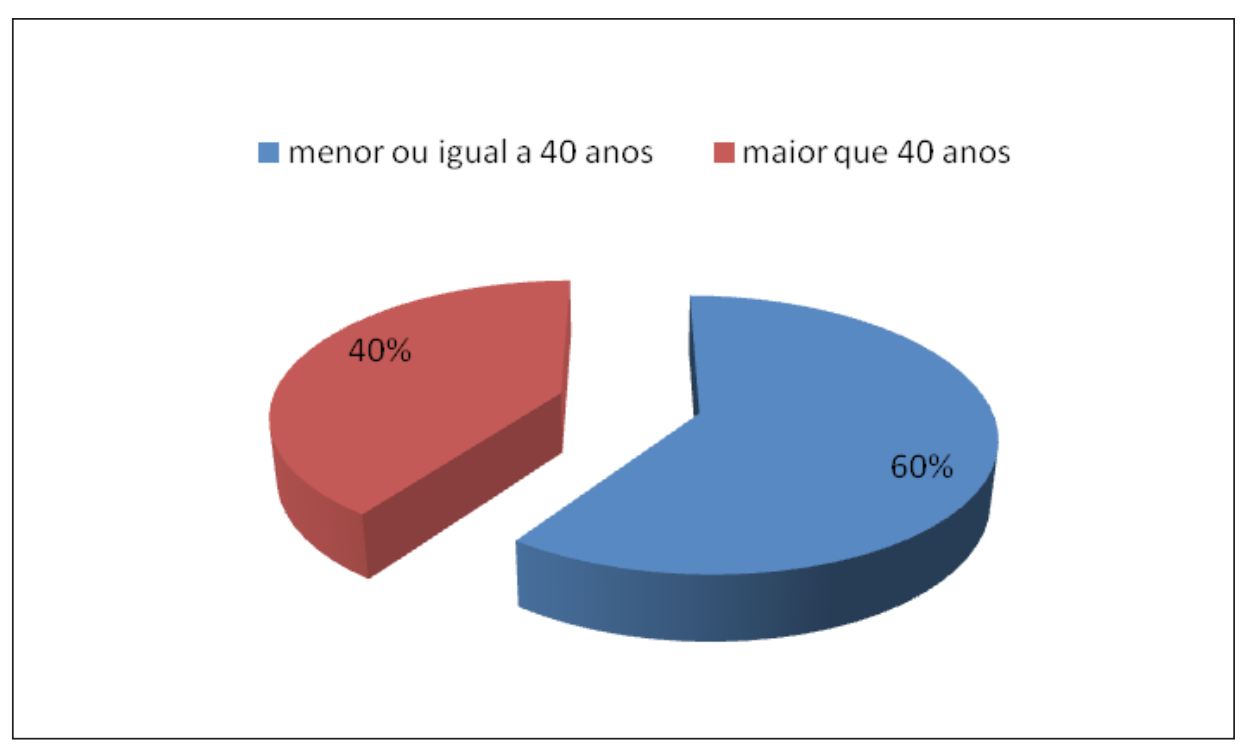

Figura 1- Faixa etária das mulheres analisadas 
Das mulheres entrevistadas, 24,5\% apresentaram menarca com idade igual ou inferior aos 11 anos, levando a uma maior exposição hormonal (Figura 2).

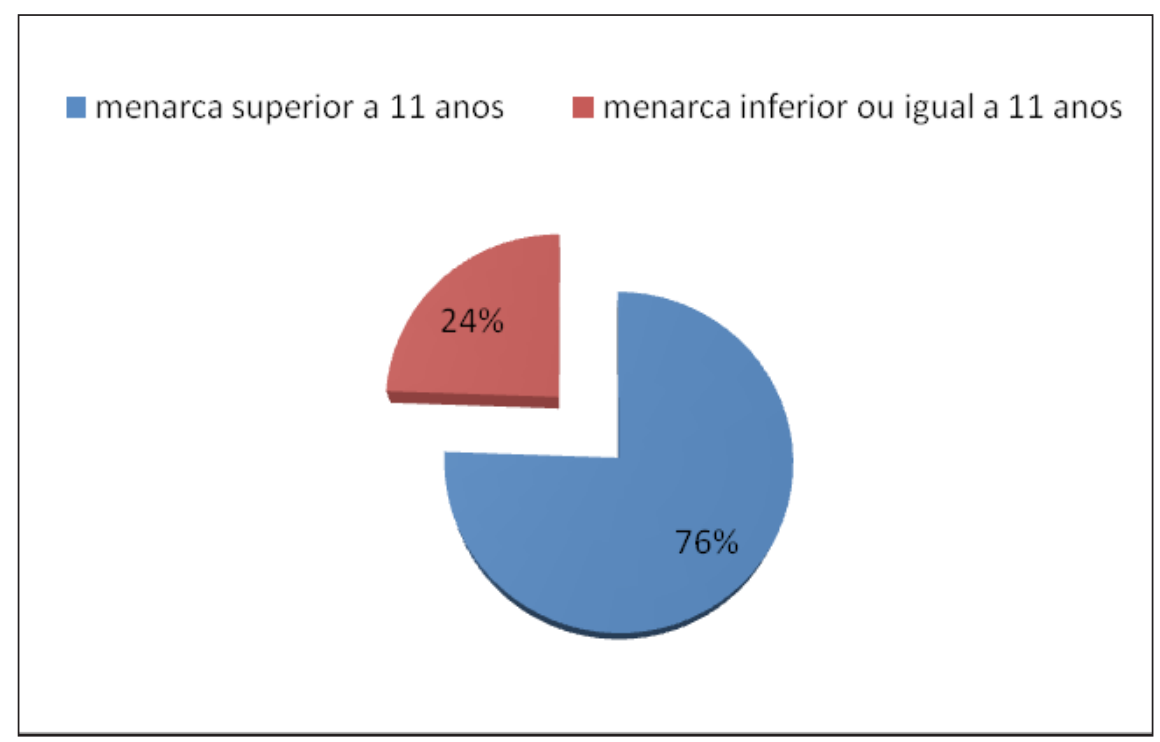

Figura 2 - Porcentagem das mulheres entrevistadas que apresentaram menarca

Das 266 mulheres apenas 36\% são nulíparas (Figura 3). Sendo que uma pequena parte das mulheres que já tiveram filhos, nunca amamentaram (10,5\%).

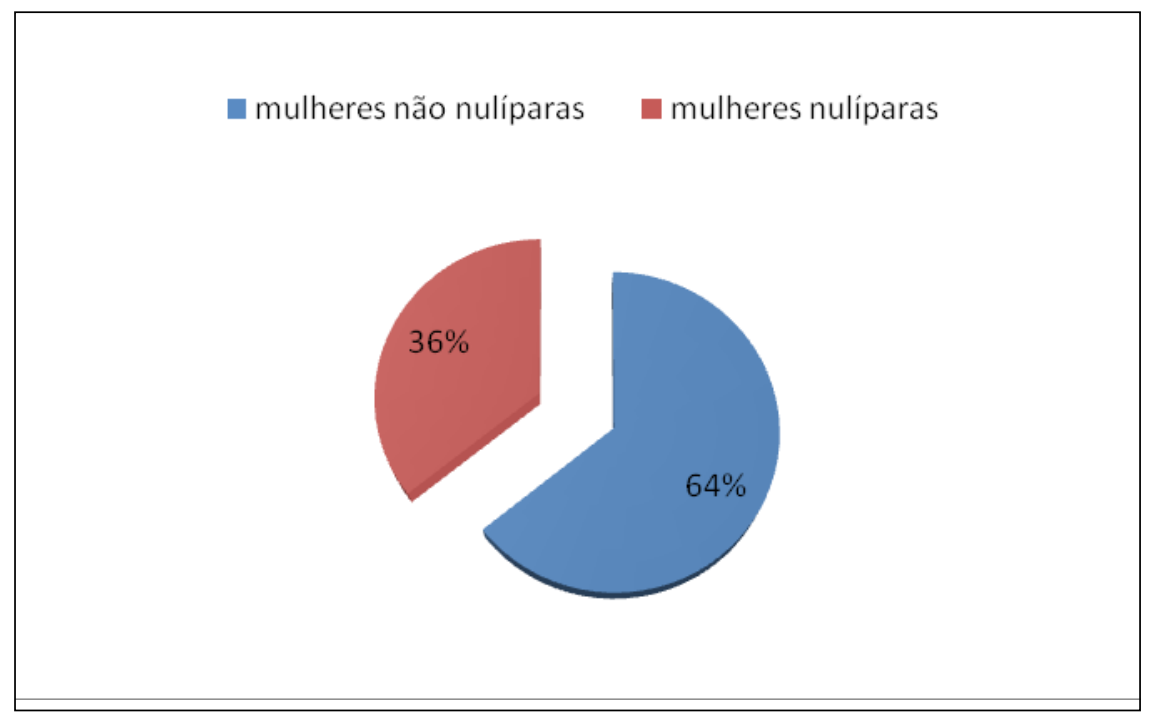

Figura 3 - Porcentagem de mulheres nulíparas 
História familiar de câncer de mama em parentes de primeiro grau foi presente em 23\% dos casos (Figura 4).

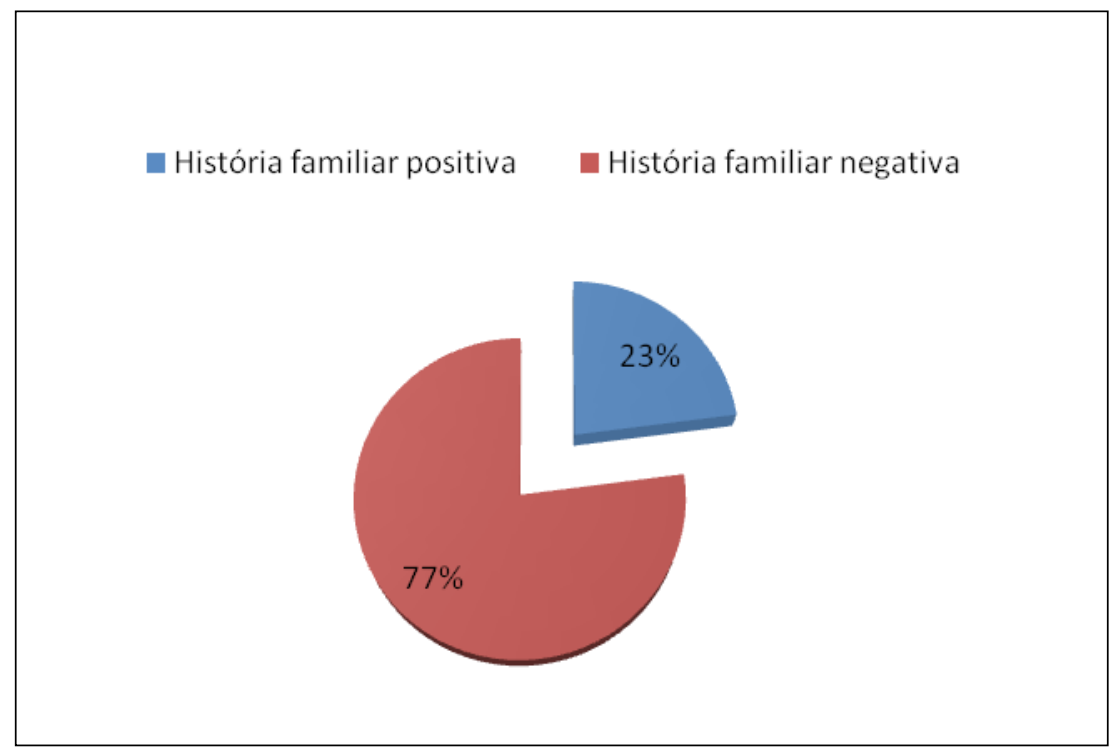

Figura 4 - Porcentagem de história familiar de câncer de mama

A terapia de reposição hormonal foi observada em uma pequena porcentagem das mulheres entrevistadas, cerca de 6\% apenas das mulheres (Figura 5).

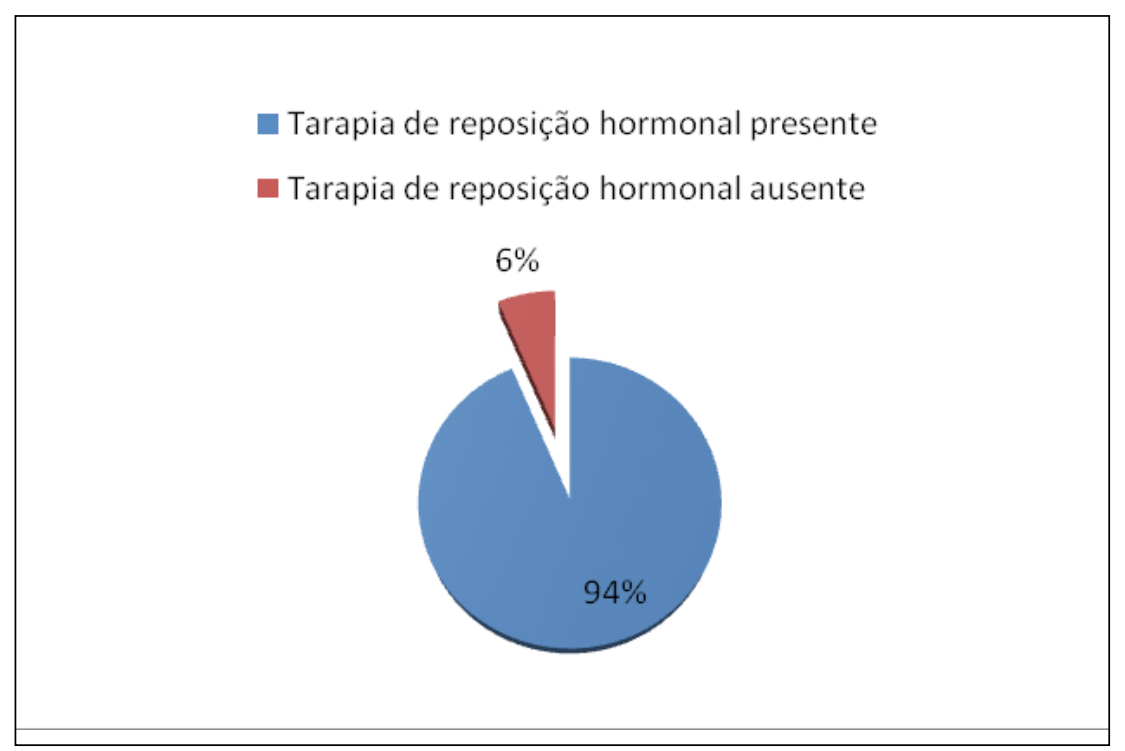

Figura 5 - Porcentagem de mulheres que realizaram terapia de reposição hormonal 


\section{Discussão}

Os resultados encontrados na pesquisa refletem a realidade encontrada pelas mulheres, no que diz respeito ao câncer de mama. Dentre os fatores de risco observados, a idade avançada foi o que mais chamou a atenção, não somente pelos números como também por ser um fator de risco não-modificável com alta relação com o câncer de mama.

Arelação de nulíparas com o câncer de mamaé considerável e constitui outras características importantes, como o desconhecimento dessa população na realização do auto-exame das mamas. Encontramos no estudo valores que ultrapassaram um pouco a média encontrada em outros estudos, cerca de 15\% a mais (Junior et al, 2006).

A base genética do câncer de mama é fator de risco indiscutível na atualidade. Muito se pesquisa sobre a relação gênica e tenta-se determinar suas alterações no maior acometimento pelo câncer de mama. Sendo assim, a história familiar de câncer de mama se faz importante em qualquer abordagem de triagem de mulheres para esse tipo de câncer. Segundo alguns autores, este fator se faz valioso no rastreio do câncer de mama. Este estudo está de acordo com a literatura, achando história familiar positiva em $23 \%$ das entrevistadas (Batiston et al, 2011).

Mais da metade das mulheres (54\%) desconhecem os fatores de risco para o câncer de mama e desta forma, não os controlam, na tentativa de reduzir tamanha incidência do mesmo (Batiston et al, 2011).

\section{Conclusão}

A promoção em saúde e qualidade de vida da mulher devem ser prioritárias em relação ao câncer de mama na mulher. $\mathrm{O}$ conhecimento da população feminina sobre os fatores que aumentam o risco desta temida doença são de extrema importância para a detecção precoce e possível abordagem terapêutica menos agressiva. Desta forma, se faz possível melhorar a atenção à saúde das mulheres com o real conhecimento dos fatores de risco para o câncer de mama e com medidas de intervenção adequada para esses fatores.

O estudo limitou-se a um pequeno número de mulheres, podendo ser estendido ao longo do tempo. 


\section{Bibliografia}

Adami HO et al (2001). Primary and secondary prevention in the reduction of cancer morbidity and mortality. Eur J Cancer; 37 Suppl 8: S118-27.

Batiston AP et al. (2011). Conhecimento e prática sobre os fatores de risco para o câncer de mama entre mulheres de 40 a 69 anos. Revista brasileira de saúde materno infantil. 11 (2): 163-171.

Carvalho CMRG et al. (2009). Prevenção de câncer de mama em mulheres idosas: uma revisão. Revista brasileira de enfermagem. 62 (4): 579-582.

Felden JBB \& Figueiredo ACL. (2011). Distribution of body fat and breast cancer: a case-control study in the South of Brazil. 16 (5): 2425-2433.

González-Robledo LM et al (2010). Acciones gubernamentales para la detección temprana del cáncer de mama en América Latina. Retos a futuro. Salud pública de méxico. 52(6):533-543.

Graham AC (2005). Epidemiology and prevention of breast cancer. Cancer Epidemiol Biomarkers Prev; 14:768-72.

Instituto Nacional de Câncer (2009). Estimativa 2010: incidência de câncer no Brasil. Rio de Janeiro: Instituto Nacional de Câncer.

Lima ALP et al (2011). Opportunistic screening for breast cancer among young women in Maranhão State, Brazil. Cad. Saúde Pública, 27(7): 1433-1439.

Piato S \&Piato JRM (2006). Doenças da mama. 1a ed. Rio de Janeiro: Revinter.

Piato S (2002). Tratado de Ginecologia. 2a ed. São Paulo: Artes Médicas.

Sclowitz ML et al (2005). Condutas na prevenção secundária do câncer de mama e fatores associados. Rev Saúde Pública, 39(3):340-9.

Torres MEA et al (2008). "Vai lá, tira a roupa... e... pronto...": o acesso a consultas ginecológicas em Belo Horizonte, MG. Rev. Bras. Est. Pop. 25 (1): 49-69.

World Cancer Research Fund (WCRF)/American Institute for Cancer Research (2007). Food, nutrition, physical activity, and the prevention of cancer: a global perspective. Washington DC: American Institute for Cancer Research. 